\title{
Effets de trois types de fertilisants sur les paramètres végétatifs et productifs du sorgho en Haïti
}

\author{
Junior ARISTIL \\ Centre de Recherche Interdisciplinaire pour la Vulgarisation Agricole et le Développement Local \\ (CREIVADEL), Université Notre Dame d'Haïti, Faculté d'Agronomie, BP: HT 8110, Redon, \\ Torbeck, Sud d'Haïti. E-mail: junior2aris@gmail.com
}

\section{RESUME}

Le sorgho (Sorghum bicolor. L) est la troisième céréale la plus consommée en Haïti. Il est cultivé dans presque tous les départements du pays y compris le Sud. Mais l'indisponibilité et de rapides augmentations du prix de l'engrais azoté (urée) sur le marché local portent à s'interroger sur de possibles solutions alternatives. L'objectif de cette étude est d'évaluer l'effet de 3 types de fertilisants chimique et biologiques (drêche, urée et compost) sur les paramètres végétatifs et productifs d'une variété de sorgho (Papesèk) dans le Sud d'Haïti. Durant la période allant de janvier à avril 2018, un dispositif Carré Latin a été mis en place pour tester la variété. Les données collectées ont été traitées avec le logiciel SPSS. Les résultats ont révélé que la masse panicule varie de 31 à 49 grammes par traitement. Le rendement en grain varie de 1,91 à 2,88 t/ha. La drêche $(2,88 \mathrm{t} / \mathrm{ha})$ a été significativement plus productive que la parcelle témoin $(\mathrm{p}<0,05)$. Mais aucune différence significative n'a été observée entre la drêche (2,88 t/ha), l'urée $(2,58 \mathrm{t} / \mathrm{ha})$ et le compost $(2,53 \mathrm{t} / \mathrm{ha}) \quad(\mathrm{p}>0,05)$. De pareils résultats suggèrent que la drêche peut facilement substituer l'urée sur les parcelles de sorgho des agriculteurs d'Haïti.

(C) 2019 International Formulae Group. All rights reserved

Mots clés: Sorgho, fertilisant, urée, drêche, compost, Haïti.

\section{Effect of three fertilizer types on vegetative and productive parameters of sorghum in Haiti}

\begin{abstract}
Sorghum (Sorghum bicolor. L) is the third most consumed cereal in Haiti. It is cultivated in all districts of Haiti including the South. But the unavailability and the often increasing of mineral fertilizer price such as urea on Haitian markets incite to develop alternative solution. This research is aimed to evaluate the effects of three fertilizer types (drêche, urea and compost) on vegetative and productive parameters of one sorghum variety (Papèsèk) in the southern district of Haiti. From January to April 2018, a dispositive of Latin square (4 $\mathrm{x} 4$ : lines $\mathrm{x}$ columns) was used to test the variety. Collected data were submitted to statistical analysis using IBM SPSS. Results revealed that panicle weight were ranging from 31 to 49 grams per treatment. Sorghum grain yield results were ranging from 1.91 to $2.88 \mathrm{t} / \mathrm{ha}$. Drêche treatment $(2.88 \mathrm{t} / \mathrm{ha})$ was significantly more productive than witness plot (no fertilization) $(\mathrm{P}<0.05)$. Even so, no significant difference was observed among drêche $(2.88 \mathrm{t} / \mathrm{ha})$, urea $(2.58 \mathrm{t} / \mathrm{ha})$ and compost $(2.53 \mathrm{t} / \mathrm{ha})$ for grain yield $(\mathrm{P}>0.05)$. These results suggest that urea could be easily substituted by drêche as fertilizer on Haitian farmer sorghum plots.
\end{abstract}

(C) 2019 International Formulae Group. All rights reserved

Keywords: Sorghum, fertilizer, drêche, compost, urea, Haiti. 


\section{INTRODUCTION}

Le sorgho (Sorghum bicolor. L) est l'une des céréales les plus cultivées dans le monde (Akata et al., 2017). Il représente l'aliment de base pour des millions de personnes en Afrique, Asie du sud et Amérique centrale (Akogou et al., 2018). Dans environ $90 \%$ des superficies cultivées en sorgho dans le monde, $70 \%$ se trouvent dans les pays en développement (Agossadou et al., 2018).

Le sorgho est une plante multifonctionnelle. Les tiges de certaines variétés servent à la fabrication de l'alcool, du sucre, du sirop, de bagasse ou fourrage et de l'éthanol (Oumarou et al., 2017). Le sorgho est aussi cultivé dans les pays tropicaux comme Haïti. Ses grains sont utilisés dans la consommation humaine directe et pour la fabrication de la farine et du pain (Sambe et al., 2017 ; Agossadou et al., 2018).

Le sorgho est la troisième céréale la plus cultivée et consommée après le riz et le maïs en Haïti. Il est cultivé principalement dans les régions de l'Artibonite, du Centre, de l'Ouest et du Sud d'Haïti. Les rendements en grains dans le Sud sont relativement faibles, environ 0,83 t/ha (Muleta et al., 2017). Le sorgho est intéressant du fait de sa bonne tolérance à la sécheresse qui est largement reconnue par les agriculteurs haïtiens. Il est très demandé dans divers département du pays. Il est également utilisé pour la production des boissons alcoolisées locales (Mukisa et al., 2017).

La faible productivité du sorgho a été documentée dans divers pays (Sainju et al., 2018 ; Dos Santos et al., 2017). Des facteurs comme la gestion des mauvaises herbes et densité de plantation ont été évalués dans des pays présentant les mêmes caractéristiques d'Haïti pour mesurer leurs impacts sur le rendement du sorgho (Alsaadawi et al., 2017 ; Tang et al., 2018). Mais en Haïti de pareilles études restent jusqu'à date très rares et concernent surtout les aflatoxines (Aristil et al., 2017). L'objectif de cette étude est d'évaluer l'effet de 3 types de fertilisants sur les paramètres productifs et végétatifs d'une variété de sorgho en Haïti.

\section{MATERIEL ET METHODES}

\section{Site de l'etude}

L'expérience a été conduite dans la section de Savannette au cours de la période allant de janvier à avril 2018. Savannette est une section communale des Cayes qui porte les coordonnées géographiques suivantes : $18^{\circ}$ $22^{\prime} \mathrm{N} 73^{\circ} 81^{\prime} \mathrm{O}$.

\section{Matériel végétal utilisé}

Pour la réalisation de l'expérience, la variété Papèsèk a été utilisée. Ce matériel provient du laboratoire du centre de recherches sur la bioénergie et l'agriculture durable (Chibas) de l'Université Quisqueya (Muleta et al., 2018).

\section{Dispositif expérimental et traitements}

Un dispositif en Carré Latin a été mis en place pour l'expérimentation. Ce dispositif a eu 16 unités expérimentales (UE) (lignes X colonnes: $4 \mathrm{X}$ 4). Chaque UE contient 4 lignes de 6 plantules ( 24 plantules/ UE). Les poquets ont été placés $65 \mathrm{~cm}$ x $30 \mathrm{~cm}$ entre et sur les lignes, ce qui correspond à une densité de $51282 \mathrm{p} / \mathrm{ha}$.

Quatre traitements ont été utilisés dans le cadre de cette étude. Ces traitements ont été : T0: (témoin) aucun apport de fertilisant n'a été fait; $\mathrm{T}_{1}: 5 \mathrm{t}$ de drêche/ha; $\mathrm{T}_{2}: 5 \mathrm{t}$ de compost/ha. $\mathrm{T}_{3}: 65 \mathrm{~kg}$ d'urée (46-0-0) /ha. Le compost et la drêche ont été appliqués en masse sèche 30 jours avant le semis. L'application de l'urée a été faite 45 jours après levée. Ces dates ont été ainsi choisies pour s'assurer d'une part, de la minéralisation complète et de la disponibilité de l'azote des matières organiques et minéral pour les plantes au temps convenable (Muliele et al., 2017 ; Kitabala et al., 2016).

\section{Pratiques agricoles}

Le semis a été fait à raison de 3 grains par poquet. Dix jours après levée de $50 \%$ des parcelles, une opération de dégarnissage a été faite pour réduire le nombre à 1 plante/ poquet. Deux opérations de sarclages ont été effectuées au cours de l'expérience. Ces deux opérations ont été réalisées respectivement 15 et 30 jours après levée. Ces opérations ont été 
choisies suivant l'évolution des mauvaises herbes dans l'aire experimentale. Trois opérations de fertilisation ont été faites. Seules les parcelles témoins n'ont pas été fertilisées. Deux types de fertilisants ont été utilisés: chimique et biologique. L'urée (46-0-0) a été l'unique engrais chimique minéral utilisé dans cette étude. Drêche et compost ont été les fertilisants organiques utilisés. Ces derniers ont été utilisés dans des proportions égales ( 5 t/ha). Pour réduire les attaques des bioagresseurs, l'insecticide chimique Tricel 480 EC a été appliqué. La dilution de cet insecticide a été faite comme recommandée par le fournisseur (Tricel : eau ; $1 \mathrm{ml}: 630 \mathrm{ml}$ : volume: volume). Les applications ont été faites suivant l'évolution des bioagresseurs dans l'aire experimentale (Halilou et al., 2017; Sane et al., 2018). Toutes ces opérations ont été arrêtées à $50 \%$ de la floraison des UE (Aristil, 2018).

\section{Collecte des données}

Deux catégories de données ont été collectées durant la conduite de l'expérimentation. Les données végétatives ont été enregistrées à $50 \%$ de la floraison des plantes par UE. Les données végétatives enregistrées ont été diamètre plante (DP), hauteur plante (HP) et longueur panicule (LP). DP a été enregistré à trois niveaux différents de la plante (au niveau du collet, au milieu du chaume et avant le point de fixation de la panicule). La circonférence a été calculée pour ces trois points de la plante. De cette circonférence $(\mathrm{C})$ a été déduit le diamètre par la formule : $\mathrm{DP}=\mathrm{C} / 3,14$. De même, la HP a été évaluée en mettant un ruban métrique sur le collet pour arriver jusqu'au premier nœud de la panicule. Les données productives ont été collectées au moment de la récolte. Les données productives enregistrées ont été : masse panicule (MP) et le rendement (Rdt). La MP a été évaluée à $13 \%$ d'humidité. Une balance de marque Fisher a été utilisée pour évaluer MP. Le rendement de chaque UE a été calculé via la formule:

$$
\operatorname{Rdt}(\mathrm{t} / \mathrm{ha})=0,051282 \times \mathrm{MP}(\mathrm{g}) .
$$

Toutes ces données ont été collectées sur un échantillon de 3 plantes par UE.

\section{Analyse statistique}

Les données collectées ont été soumises à l'analyse des variances (ANOVA) en utilisant les lignes comme fixes et les traitements comme variables. Le test Welsch et Gabriel a été utilisé pour évaluer le niveau d'homogénéité entre les variables. Ces tests ont été réalisés à un niveau de confiance de 95\%. Toutes ces analyses ont été réalisées en utilisant le logiciel IBM SPSS, version 22.0 (Corp, 2013).

\section{RESULTATS}

\section{Analyse des variances des paramètres}

Sur le Tableau 1 et la Figure 1 sont notés les résultats de l'ANOVA des données collectées. Tous les paramètres présentent des différences significatives entre les traitements $(\mathrm{p}<0,01)$.

Le DP varie entre 20 et $32 \mathrm{~mm}$. Le diamètre minimal a été enregistré sur T0 $(20,25 \mathrm{~mm})$ alors que le diamètre maximal a été noté sur (T1). Seul T3 n'a pas été significativement différent de $\mathrm{T} 1 \quad(\mathrm{p}>0,05)$. Par contre aucune difference significative n'a été détectée entre T0 et T2 (p>0,05). Soixante-quinze pourcent $(75 \%)$ des plantes des traitements ont un diamètre $<30 \mathrm{~mm}$.

La HP tourne autour de 118 et $144 \mathrm{~cm}$. La hauteur minimale a été enregistrée sur T0 $(118 \mathrm{~cm})$ alors que la hauteur maximale a été notée sur (T1). T1 a été significativement plus développé pour ce paramètre comparé aux autres traitements $(\mathrm{p}<0,05)$. T2 et T3 n'ont pas été significativement différents de $\mathrm{T} 1$ (p> 0,05 ). Pourtant T0 a été significativement moins développé comparé aux autres. Seul le traitement $\mathrm{T} 1$ a enregistré une hauteur supérieure à $140 \mathrm{~cm}$.

La LP varie entre 15 et $24 \mathrm{~cm}$. La longueur minimale a été enregistrée sur T0 $(15,20 \mathrm{~cm})$ alors que la longueur maximale a été notée sur (T1). T1 a été significativement plus développés pour ce paramètre comparé aux autres traitements $(p<0,05)$. Les résultats de l'analyse de la longueur ont révélé que les traitements ont été classés en 2 groupes. Ces groupes ont été identifiés par les lettres « a » et $« b »$. 
La MP varie entre 31 et $49 \mathrm{~g}$. La MP minimale a été enregistrée sur T0 $(31,40 \mathrm{~g})$ alors que la maximale a été notée sur (T1). T2 et T3 n'ont pas été significativement différents de T1 $(p>0,05)$. Seul le traitement T1 a enregistré une MP $>45 \mathrm{~g}$.

Sur la Figure 1 sont présentés les résultats obtenus pour le rendement des plantes. Des différences significatives ont été observées entre les résultats obtenus pour ce paramètre. Ces résultats ont révélé que les rendements obtenus pour les 4 traitements ont été classés en deux groupes. Ces groupes ont été désignés par les lettres « $\mathrm{a} »$ et $« \mathrm{~b} »$. Le rendement varie entre 1,9 à 2,9 t/ha. Le plus faible rendement a été enregistré sur T0 $(1,2$ t/ha) alors que le rendement maximal a été noté sur (T1). T1 a été plus productif que les autres traitements. T2 et T3 n'ont pas été significativement différents de $\mathrm{T} 1 \quad(\mathrm{p}>0,05)$.

Tableau 1. Résultats de l'analyse des variances des paramètres sous l'effet des trois types de fertilisants.

\section{Paramètres}

\begin{tabular}{ccccc}
\hline Traitements & $\begin{array}{c}\text { Diamètre plante } \\
(\mathrm{mm})\end{array}$ & $\begin{array}{c}\text { Hauteur plante } \\
(\mathrm{cm})\end{array}$ & $\begin{array}{c}\text { Longueur panicule } \\
(\mathrm{cm})\end{array}$ & $\begin{array}{c}\text { Masse panicule } \\
(\mathrm{g})\end{array}$ \\
\hline T0 & $20,25 \mathrm{a}$ & $118,25 \mathrm{a}$ & $15,5 \mathrm{a}$ & $31,75 \mathrm{a}$ \\
\hline $\mathrm{T} 1$ & $31,33 \mathrm{c}$ & $143 \mathrm{~b}$ & $23,28 \mathrm{~b}$ & $48,04 \mathrm{~b}$ \\
\hline $\mathrm{T} 2$ & $23,17 \mathrm{ab}$ & $136,75 \mathrm{~b}$ & $18,37 \mathrm{a}$ & $42,23 \mathrm{ab}$ \\
\hline T3 & $28,25 \mathrm{~b}$ & $134,92 \mathrm{~b}$ & $17,52 \mathrm{a}$ & $43,05 \mathrm{~b}$ \\
\hline Moyenne & 25,75 & 133,23 & 18,67 & 41,27 \\
\hline Ecart type & 8,10 & 13,90 & 4,19 & 12,56 \\
\hline Probabilité & 0,002 & 0,001 & 0,001 & 0,009 \\
\hline
\end{tabular}

T0 : témoin (sans engrais); T1 : 5 t/ha de drêche; T2 $: 5 \mathrm{t} / \mathrm{ha}$ de compost; T3 $: 65 \mathrm{~kg} /$ ha d'urée (46-0-0). Les variables de lettres différentes sont statistiquement différentes pour le test Welsch et Gabriel $(\mathrm{p}<0,05)$.

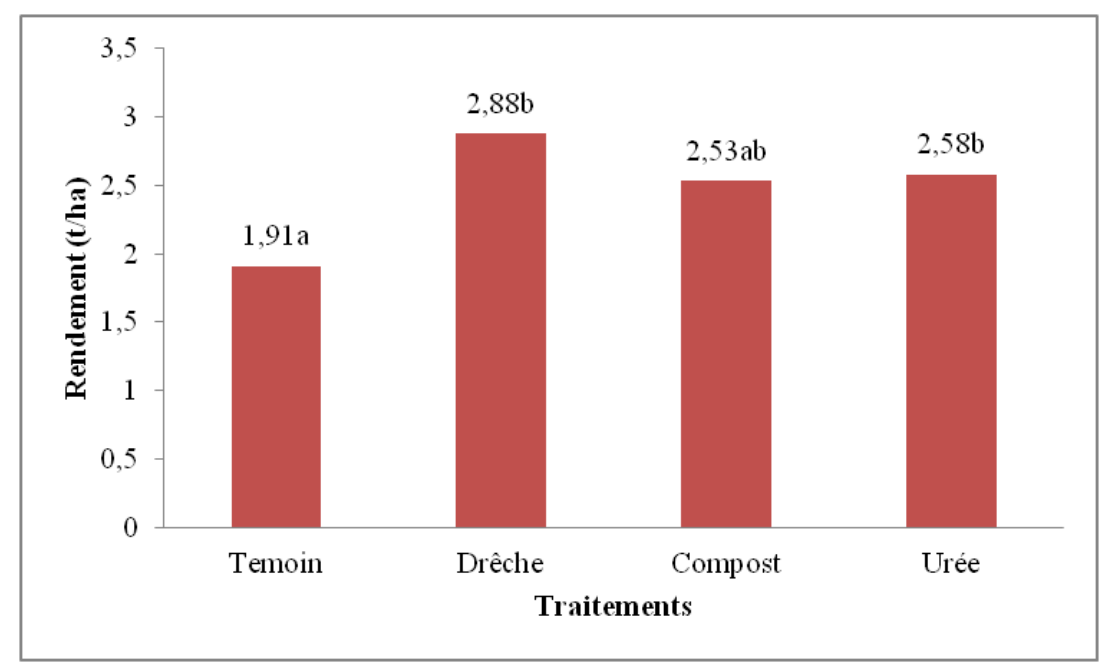

Figure 1 : Variation du rendement $(\mathrm{t} / \mathrm{ha}) \mathrm{du}$ sorgho sous l'effet des trois types de fertilisants. Les barres de mêmes lettres ne sont pas significatives ( $p>0,05)$. 


\section{DISCUSSION}

Le sorgho est l'une des principales cultures céréalières d'Haïti. Il est la troisième céréale en termes de superficie emblavée. Cette céréale est plantée dans presque tous les départements du pays y compris le Sud. Ses grains entrent dans la fabrication de la farine. Les feuilles sont également utilisées dans l'alimentation animale. Les agriculteurs haïtiens font face souvent à de sérieux problèmes résultant de l'indisponibilité et parfois du coût excessif des fertilisants minéraux dont l'urée, sur le marché local. Pour atténuer ce problème, il devient plus qu'une impérative de trouver une solution alternative à l'urée. Ainsi l'objectif de cette étude est d'évaluer l'effet de trois types de fertilisants sur les paramètres végétatifs et productifs du sorgho en Haïti. Un total de 5 paramètres relatifs à la végétation et la production a été enregistré et soumis à des analyses statistiques en utilisant le logiciel SPSS, version 22.0. Ces paramètres enregistrés ont été : DP, HP, LP, MP et Rdt.

Le DP varie entre 20 et $32 \mathrm{~mm}$. Par contre aucune difference significative n'a été détectée entre T0 et T2 ( $>$ > 0,05). Soixantequinze pourcent $(75 \%)$ des plantes des traitements ont un diamètre $<30 \mathrm{~mm}$. Les résultats obtenus dans le cadre de cette présente recherche sont en désaccord avec les données de (Djè et al., 2007 et Allam et al., 2018). Djè et ses collègues (2007) travaillant sur l'évaluation de la diversité morphologique $\mathrm{du}$ sorgho au Nord d'ouest du Maroc ont rapporté avoir obtenu des diamètres plantes variant de $75-122 \mathrm{~cm}$. Cette difference observée entre les deux expériences peut être expliquée par les différences agroécologiques des deux pays (Maroc et Haïti).

La HP varie de 118 et $144 \mathrm{~cm}$. T1 a été significativement plus développé pour ce paramètre comparé aux autres traitements $(\mathrm{p}<$ $0,05)$. T2 et T3 n'ont pas été significativement différents de $\mathrm{T} 1$ ( $p>0,05)$. Les résultats obtenus pour la HP sont similaires à ceux de (Wang et al., 2009 ; Abdou et al., 2014).

La LP varie entre 15 et $24 \mathrm{~cm}$. Les résultats de l'analyse de la longueur ont révélé que les traitements ont été classés en 2 groupes. Les résultats obtenus confirment les données d'Abdi et al. (2002).

La MP varie entre 31 et $49 \mathrm{~g}$. La MP minimale a été enregistrée sur T0 $(31,4 \mathrm{~g})$ alors que la maximal a été noté sur (T1). T2 et T3 n'ont pas été significativement différent de $\mathrm{T} 1$ ( $\mathrm{p}>0,05)$. Seul le traitement $\mathrm{T} 1 \mathrm{a}$ enregistré une MP > $45 \mathrm{~g}$. Ces résultats supportent ceux d'Ezeaku et Mohammed, (2006).

Le rendement des traitements varie entre 1,9 à 2,9 t/ha. Le plus faible rendement a été enregistré sur T0 (1,2 t/ha) alors que le rendement maximal a été noté sur (T1). T1 a été plus productif que les autres traitements. $\mathrm{T} 2$ et T3 n'ont pas été significativement différents de $\mathrm{T} 1(\mathrm{p}>0,05)$. Ces résultats se différent de ceux de Deb et al. (2004). Le faible rendement obtenu avec T0 peut être expliqué par la faible proportion d'éléments minéraux dans le sol comme l'azote, potassium et phosphate résultant de la décomposition rapide de la matière organique dans les zones tropicales (Traoré et al., 2012). La matière organique ameublit le sol et permet une bonne alimentation hydrique et minérale du sorgho qui est associé (Bazongo et al., 2015).

\section{Conclusion}

Le sorgho est la troisième céréale la plus consommée en Haïti après le riz et le maïs. Il est cultivé dans presque tous les départements d'Haïti. Le Sud est l'un des plus importants. Dans le cadre de cette recherche, il a été objectivé d'évaluer l'effet de trois types de fertilisants sur les paramètres productifs du sorgho en Haïti. Les résultats ont révélé que le rendement des traitements varie de 1,9 à 2,9 t/ha. Aucune difference significative n'a été observée entre les rendements obtenus avec la drêche et celui de l'urée. Ces résultats supposent que la drêche peut facilement substituer l'urée et le compost dans la fertilisation du sorgho en Haïti. Des études supplémentaires sont nécessaires pour confirmer ces résultats. 


\section{CONFLIT D'INTERETS}

L'auteur déclare qu'il n'existe aucun conflit d'intérêts.

\section{REMERCIEMENTS}

Profond remerciement à Exilier Berlineda pour sa contribution dans la mise en place et la conduite de l'expérimentation.

\section{REFERENCES}

Abdi A, Bekele E, Asfaw Z, Teshome A. 2002. Patterns of morphological variation of sorghum (Sorghum bicolor (L.) Moench) landraces in qualitative characters in North Shewa and South Welo, Ethiopia. Hereditas., 137(3): 161172. DOI: https://doi.org/10.1034/j.16015223.2002.01604.x

Abdou MM, Mayaki ZA, Lamso ND, Seybou DE, Ambouta, J M K. 2014. Productivité de la culture du sorgho (Sorghum bicolor) dans un système agroforestier à base d'Acacia senegal (L.) Willd. au Niger. Journal of Applied Biosciences, 82(1): $\quad 7339-7346 . \quad$ DOI: http://dx.doi.org/10.4314/jab.v82i1.2

Agossadou MM, Sêgla SS, Adégbidi, AA, Kayodé, PA. 2018. Connaissances Paysannes Et Prédisposition À Adopter Une Innovation En Agro-Alimentaire: Cas Du Décorticage Mécanique Et De La Fortification En Fer Du Sorgho Dans Le Nord-Bénin. European Scientific Journal, ESJ., 14(24). DOI : http://dx.doi.org/10.19044/esj.2018.v14n 24p432

Akata EA, Diatta C, Faye JM, Diop A, Maina F, Sine B, Cisse, N. 2017. Combining ability and heterotic pattern in West African sorghum landraces. African Crop Science Journal., 25(4) : 491-508. DOI: http://dx.doi.org/10.4314/acsj.v25i4.7

Akogou FUG, Kayodé APP, den Besten H MW, Linnemann AR, Fogliano V. 2018. Effects of processing and storage on the stability of the red biocolorant apigeninidin from sorghum. LWT., 90 : 592-597.

DOI : https://doi.org/10.1016/j.lwt.2017.12.071

Allam A, Tirichine A, Madani H, Benlamoudi W. 2018. Variabilité morphologique du sorgho (Sorghum Bicolor L. Moench), cultivé dans la Vallée d'Oued Righ (SudEst algérien). Lebanese Science Journal., 19(1): $10 . \quad$ DOI: http://dx.doi.org/10.22453/LSJ019.1.010-018

Alsaadawi IS, Tawfiq AA, Malih H M. 2017. Effect of Allelopathic Sorghum Mulch on Growth and Yield of Faba Bean (Vicia faba) and Companion Weeds. Journal of Plant Protection, 12 : 123127.

Aristil J. 2018. Integrated approaches to agriculture in developing country: Haiti. $\mathrm{PhD}$ thesis, University of Milan, Milan, $\mathrm{p}$ 174.

Aristil J, Venturini G, Spada A. 2017. Occurrence of Toxigenic Fungi and Aflatoxin Potential of Aspergillus spp. Strains Associated with Subsistence Farmed Crops in Haiti. J. Food Prot., 80(4): 626-631.

Bazongo P, Traore K, Traore O, Yelemou B, Sanon KB, Kabore S, Hien V, Nacro BH. 2015. Influence des haies de Jatropha sur le rendement d'une culture de sorgho (Sorghum vulgare) dans la zone Ouest du Burkina Faso: cas du terroir de Torokoro. Int. J. Biol. Chem. Sci., 9(6): 2595-2607. DOI:

http://dx.doi.org/10.4314/ijbcs.v9i6.7

Corp IBM. 2013. IBM SPSS statistics for windows, version 22.0. Armonk, NY: IBM Corp.

Deb UK, Bantilan MCS, Roy AD, Rao PP. 2004. Global sorghum production scenario. Sorghum genetic enhancement: research process, dissemination and impacts, (2): 21-38.

Djè Y, Heuertz M, Ater M, Lefebvre C, Vekemans X. 2007. Évaluation de la diversité morphologique des variétés traditionnelles de sorgho du Nord-ouest du Maroc. Biotechnol Agron Soc., 11(1): 39-46.

Dos Santos CLR, Alves GC, de Matos Macedo AV, Giori FG, Pereira W, Urquiaga S, Reis VM. 2017. Contribution of a mixed inoculant containing strains of Burkholderia spp. and Herbaspirillum ssp. to the growth of 
three sorghum genotypes under increased nitrogen fertilization levels. Appl Soil Ecol., 113: 96-106. DOI: https://doi.org/10.1016/j.apsoil.2017.02.0 08

Ezeaku IE, Mohammed SG. 2006. Character association and path analysis in grain sorghum. Afr J Biotechnol., 5:14

Halilou H, Kadri A, Karimou I. 2017. Gestion intégrée du mildiou du mil en station au centre régional de recherche agronomique de Maradi (CERRA/Maradi) au Niger. Int. J. Biol. Chem. Sci., 11(6): 2704-2712. DOI : https://dx.doi.org/10.4314/ijbcs.v11i6.12

Kitabala MA, Tshala UJ, Kalenda MA, Tshijika IM, Mufind KM. 2016. Effets de différentes doses de compost sur la production et la rentabilité de la tomate (Lycopersicon esculentum Mill) dans la ville de Kolwezi, Province du Lualaba (RD Congo). Journal of Applied Biosciences, 102(1) : 9669-9679. DOI: http://dx.doi.org/10.4314/jab.v102i1.1

Mukisa IM, Byaruhanga YB, Muyanja CM, Langsrud T, Narvhus JA. 2017. Production of organic flavor compounds by dominant lactic acid bacteria and yeasts from Obushera, a traditional sorghum malt fermented beverage. Food science \& nutrition., 5(3): 702-712. DOI: https://doi.org/10.1002/fsn3.450

Muleta KT, Pressoir G, Morris GP. 2018. Optimizing genomic selection for a sorghum breeding program in Haiti: a simulation study. G3: Genes, Genomes, Genetics., $\quad$ g3-200932. DOI: https://doi.org/10.1534/g3.118.200932

Muliele TM, Nsombo BM, Kapalay OM, Mafuka PM. 2017. Amendements organiques et dynamique de l'azote minéral dans le sol sableux de Kinshasa (RD Congo). Journal of Animal and Plant Sciences, 32(2) : 5156-5167.

Oumarou Y, Saidou AA, Madi A, Watang Zieba F, Fokou Yemata O. 2017. Perception paysanne des perturbations pluviométriques et stratégies d'adaptation dans les systèmes de culture à sorgho repiqué en zone soudano-sahélienne du Cameroun. Afrique Science, 13(4): 5065

Sainju UM, Singh H P, Singh B P, Chiluwal A, Paudel R. 2018. Soil Carbon and Nitrogen under Bioenergy Forage Sorghum Influenced by Cover Crop and Nitrogen Fertilization. Agrosystems, Geosciences \& Environment, 1:1

Sambe M, Tounkara LS, Lopy MFJS, N'diaye Y. 2017. Etude des comportements rheologiques des melanges de farine ble/sorgho sans tanins issue de trois nouvelles varietes cultivees au Senegal et mise au point de pains à base de farines composées (ble/sorgho). Agronomie Africaine., 29(1) : 69-74.

Sane B, Badiane D, Gueye MT, Faye O. 2018. Évaluation de l'efficacité biologique d'extrait de neem (Azadirachta indica Juss.) comme alternatif aux pyréthrinoïdes pour le contrôle des principaux ravageurs du cotonnier (Gossypium hirsutum L.) au Sénégal. Int. J. Biol. Chem. Sci., 12(1): 157-167. DOI https://dx.doi.org/10.4314/ijbcs.v12i1.12

Tang C, Sun C, Du F, Chen F, Ameen A, Fu T, Xie GH. 2018. Effect of plant density on sweet and biomass Sorghum production on semiarid marginal land. Sugar Tech., 20(3): 312-322. DOI: 10.1007/s12355-017-0553-3

Traoré M, Nacro HB, Tabo R, Nikièma A, Ousmane H. 2012. Potential for agronomical enhancement of millet yield via Jatropha curcas oilcake fertilizer amendment using placed application technique. Int. J. Biol. Chem. Sci., 6(2):808-819.

DOI: http://dx.doi.org/10.4314/ijbcs.v6i2.23

Wang ML, Zhu C, Barkley NA, Chen Z, Erpelding JE, Murray SC, Yu J. 2009. Genetic diversity and population structure analysis of accessions in the US historic sweet sorghum collection. Theor Appl Genet., 120(1): 13-23. DOI: 10.1007/s00122-009-1155-6. 